\section{Cahiers de Narratologie}

Analyse et théorie narratives

13 | 2006

Nouvelles approches de l'intertextualité

\title{
Du reportage au roman ou la greffe au cSur d'une poétique narrative
}

\section{(2) OpenEdition}

Journals

Édition électronique

URL : http://journals.openedition.org/narratologie/356

DOI : $10.4000 /$ narratologie.356

ISSN : 1765-307X

Éditeur

LIRCES

\section{Référence électronique}

"Du reportage au roman ou la greffe au cSur d'une poétique narrative », Cahiers de Narratologie [En ligne], 13 | 2006, mis en ligne le 11 septembre 2006, consulté le 15 novembre 2019. URL : http:// journals.openedition.org/narratologie/356 ; DOI : 10.4000/narratologie.356

Ce document a été généré automatiquement le 15 novembre 2019.

\section{(c) (i) $\odot$}

Cahiers de Narratologie - Analyse et théorie narratives est mis à disposition selon les termes de la licence Creative Commons Attribution - Pas d'Utilisation Commerciale - Pas de Modification 4.0 International. 


\section{Du reportage au roman ou la greffe au cSur d'une poétique narrative}

1 Dans sa maison de Saint-Cyr-sur-Morin, le béret écossais vissé sur la tête et le perroquet Dagobert juché sur l'épaule, Pierre Mac-Orlan évoquait volontiers avec ses visiteurs les quinze années au cours desquelles il avait voyagé à travers l'Europe et l'Afrique du Nord, en qualité de reporter pour le compte de L'Intransigeant ou de ParisSoir. Pendant l'entre-deux guerres, il a été correspondant de L'Intransigeant en Rhénanie occupée (1918-1919). Par la suite, il a interviewé Mussolini, avant de couvrir pour ParisSoir l'élection de Hitler à Berlin, en 1932. A partir du milieu des années vingt, le journaliste s'est essayé à la chronique judiciaire. Il rendit compte du procès des «Pirates de l'avenue du Rhum » dans les colonnes de L'Intransigeant du 28 octobre au 5 novembre 1924. En 1934, c'est pour le quotidien Le Journal qu'il se rend à Londres et à Brighton, où, à la découverte d'une malle contenant le tronc d'une femme enceinte a succédé celle d'une autre malle renfermant le corps démembré d'une seconde femme, tuée avant la première. Mac Orlan enquête sur ce double assassinat. Il décrit les lieux du crime et interroge l'entourage du présumé assassin de la victime retrouvée dans la malle $\mathrm{n}^{\circ} 2$. A ce journalisme d'investigation s'est ajouté un journalisme de témoignage mêlant impressions, descriptions et réflexions éclairantes, avec, d'une part, les articles sur la légion étrangère française et espagnole installée au Maroc, et, d'autre part, ceux consacrés aux bataillons disciplinaires d'Afrique établis dans le sud tunisien.

2 Ces articles ont été ensuite réunis et publiés sous la forme de reportages ${ }^{1}$, à une époque où ce genre, très prisé par le grand public, répond à une attente de dépaysement et de vérité documentaire, tout en accédant au statut d'œuvre littéraire grâce à des écrivains de talent comme Blaise Cendrars, Joseph Kessel, Roland Dorgelès, ou Paul Morand. De surcroît, cet écrivain polygraphe, auteurs de chansons, de poèmes, de contes et de souvenirs, a également publié une quinzaine de romans où se croisent des figures récurrentes, archétypiques et attachantes, comme celles du malfrat pressé par la misère, du légionnaire, du forban ou de la fille de bars à matelots. De ce dernier ensemble se détachent cinq romans ${ }^{2}$ qui empruntent partiellement leur substance aux reportages précédemment nommés ${ }^{3}$. 
3 Ainsi se constituent cinq paires d'ouvrage rapprochés en vertu d'un rapport de filiation, puisque chaque récit factuel donne naissance à un récit fictionnel : Les Pirates de l'avenue du Rhum (1925) / Dinah Miami (1928); Images sur la Tamise (1925) / Quartier réservé (1932) ; Légionnaires (1930) / La Bandera (1931) ; Le Bataillon de la mauvaise chance (1933) / Le Camp Domineau (1937); Le Mystère de la malle $n^{\circ} 1$ (1934) / Le Tueur $n^{\circ} 2$ (1935). Les reportages ont fourni au romancier une abondante provende de sujets, d'images et de personnages que l'on retrouve sous des formes diverses dans les romans. Ces prélèvements ressortissent à une intertextualité interne ou restreinte, nommée intratextualité par les narratologues ${ }^{4}$. En effet, les textes circulent au sein d'un espace autographe. Ce sont les modalités et les effets de cette migration intratextuelle et clandestine que nous envisagerons dans cette étude, après avoir montré que le reportage mac orlanien, discours sur le monde, est aussi travaillé par des références à des textes allographes, par des formes d'inscription citationnelle. Nous nous emploierons donc à mesurer l'apport de l'hypotexte journalistique à la création romanesque. Nous tenterons de saisir les corrélations qui se tissent entre les régimes factuel et fictionnel du récit en empruntant, fort modestement, la voie tracée par Gérard Genette qui, dans Fiction et diction, engageait les narratologues à s'inspirer de l'exemple des formes narratives, lesquelles « traversent allègrement la frontière entre fiction et non-fiction $»^{5}$.

Les pratiques intertextuelles à l'œuvre dans le reportage

Rédigé à la suite d'un voyage, d'une enquête ou d'une immersion dans un milieu social spécifique (les légionnaires ou les bataillonnaires), le reportage mac orlanien mêle l'information judiciaire, historique et sociologique au témoignage vécu. Il explore des milieux sociaux mal connus ou ignorés du grand public. Cette orientation référentielle va de pair avec une visée documentaire et didactique. Les informations collectées sont, dans l'ensemble, rapportées au seul travail d'investigation du journaliste. Mais, dans le second chapitre des Pirates de l'avenue du Rhum, Mac Orlan cite un extrait des instructions adressées par les armateurs aux capitaines chargés d'organiser la vente de l'alcool, à la limite des eaux territoriales américaines. La consigne reproduite mentionne l'interdiction d'accepter un passager à bord du vapeur. Signalée par les guillemets, cette citation est chargée d'une portée pragmatique. Elle fournit une information qui guide un lecteur investi de la fonction de juge par procuration, dans une affaire à propos de laquelle le tribunal de Brest n'avait pas encore rendu son verdict lors de la publication du reportage. La citation instruit indirectement le lecteur de la responsabilité du capitaine du Mulhouse, lequel a enfreint la consigne de son armateur en accueillant à bord de son cargo trois personnes du Patara. Dès lors, l'intertexte se révèle l'un des éléments du système d'appel en fonction duquel le reporter requiert la participation du lecteur.

Une autre forme explicite d'intertextualité, la référence, est mise à contribution dans le troisième chapitre. La mention d'un article publié dans un journal américain apporte une caution scientifique à l'analyse de l'efficacité des mesures prises par les autorités américaines. La référence est complétée par une brève synthèse des données numériques procurées par l'article: "Une note parue récemment dans The Chicago Tribune du 22 octobre 1924 indique, au point de vue de la répression de la contrebande de l'alcool, les résultats obtenus par l'autorité. Dans les trois années de prohibition on a arrêté 177000 individus, et le total des années de prison distribuées s'élève au chiffre imposant de $7000^{6} »$. Fenêtre ouverte sur la documentation réunie par le reporter, la 
référence concourt à l'effet d'authenticité propre à l'écriture du reportage, tout en satisfaisant «le fétichisme du concret $^{7}$ » de ce lectorat. Mais, de manière plus inattendue, la référence au récit factuel met en évidence les potentialités littéraires de l'épisode central de ce reportage, en l'occurrence l'abordage du Mulhouse. La mention des sources utilisées pour relater cette scène, «le rapport du capitaine Ferrero et le récit saisissant de $\mathrm{M}$. Walter James Kimpton ${ }^{8}$ ", s'accompagne d'un commentaire qui engage le lecteur à interroger les frontières qui séparent les régimes factuel et fictionnel du récit.

6 Cette pratique intertextuelle assume également une fonction argumentative. Elle illustre et motive la mise en évidence d'une relation entre le particulier et le général, entre l'individuel et le typique. C'est à la faveur d'une allusion aux récits factuels du capitaine Johnson, historiographe des flibustiers du XVIII ${ }^{2}$ è siècle, que l'humour noir, le rire cruel et cynique des pirates des temps modernes est rapproché des facéties des corsaires : «En ceci comme en beaucoup d'autres choses, les mauvais garçons de la "Rhum navy " (la Flotte du Rhum), se rattachent singulièrement à l'esprit de corps des compagnons de Flint [...] Ceux qui, aujourd'hui, prennent à tâche de les imiter, sont exactement de la même essence ${ }^{9}$ ». Comme l'a montré Michel Collomb ${ }^{10}$, ce mouvement pendulaire de la réflexion est l'une des propriétés du reportage. Il ressortit manifestement à une stratégie de valorisation du fait divers.

7 De surcroît, un second intertexte, proprement littéraire, se greffe sur le premier. Plus précisément, les faits authentiques relatés par Johnson au XVIII ${ }^{\text {ème }}$ siècle sont mis en regard de la geste ubuesque: "Cette comédie, d'un comique assez brutal, rappelle étrangement les scènes les plus féroces d'Ubu Roi». Ainsi le portrait des pirates de la " rum-row » est-il esquissé à partir d'un double prisme littéraire qui estompe, puis brouille, efface partiellement les contours du référent réel. Interposant un double écran, légendaire et grotesque, entre les personnages authentiques mis en scène et le lecteur, l'intertexte crée un effet de sfumato. Il revêt d'un aspect vaporeux les personnages comme les situations. Dans ce reportage, il n'est pas rare que le journaliste prête sa voix au romancier, pour qui l'imaginaire littéraire constitue la voie d'accès la plus fructueuse à la connaissance de ces nouveaux pirates. Ces forbans se révèlent être des « hommes parfaitement conformes à tout ce que nous pouvons exiger de notre imagination, quand elle s'excite à la lecture des romans de Stevenson, des Vies imaginaires de Marcel Schwob, et des petits livres de colportage qui font entrer la peur dans les maisons isolées ${ }^{11}$ ».

8 La référence n'est pas une simple réminiscence. Si elle ne relève pas d'une stratégie destinée à contourner la difficulté à dire le réel, comme on peut le voir dans le récit de voyage, elle fait office de médiation. Dans Le Bataillon de la mauvaise chance, la description de Carthage est mêlée à des souvenirs de lecture de Salammbô. L'évocation de la pénurie d'eau est suivie d'une allusion aux souffrances que la soif inflige aux soldats de Matho. Le référent littéraire est comparé au référent réel pour suggérer l'immobilité d'une société figée dans ses traditions. Le lecteur peut légitimement s'interroger sur la pertinence heuristique du rapprochement opéré entre l'antiquité fictionnelle et la modernité de la "chose vue». A ces relations de médiation et de comparaison s'en ajoute une autre, susceptible de compléter la typologie des pratiques intertextuelles dans les récits de voyage établie par Christine Montalbetti ${ }^{12}$. À la faveur de ce qui s'apparente à une hallucination visuelle ou à une construction onirique, le personnage romanesque, en l'espèce Narr'Havas, le prince des Numides, est associé à 
un contexte militaire moderne : «[...] je me représentais Narr'Havas vêtu du burnous noir des boujliouiles italiens. Il précédait sa bande de partisans armés du curieux mousqueton des savaris dont la baïonnette fixée au canon par une charnière se replie le long de l'arme ${ }^{13} »$. On pourrait nommer relation d'interférence cette collusion des deux univers, référentiel et fictionnel, dans la même séquence. Réduite à un syntagme nominal ( les soldats d'Hannon ${ }^{14} »$ ) cette interférence fait office de périphrase suggérant les qualités des bataillonnaires. La confusion entre l'espace réel et l'espace fictionnel est encore sensible dans les considérations du reporter sur les personnages de Salammbô. Mathô est présenté comme la simple transcription romanesque d'une personne rencontrée par Flaubert sur la route qui conduit à Carthage. Cette manifestation du "complexe de Victor Bérard", pour reprendre la formule de Christine Montalbetti, se double d'un commentaire métatextuel qui récuse implicitement l'hétérogénéité du texte littéraire et du monde : «[Salammbô] n'est pas une valeur humaine absolument vraie comme son père, comme Mathô, comme le prêtre de Tanit $[. . .]^{15}$ "

Point d'ancrage, l'intertexte est utilisé à des fins herméneutiques dans Le Mystère de la malle numéro 1 . Le troisième chapitre de ce reportage, centré sur la personnalité de l'assassin présumé, Jacq Notyre, alias Mancini, porte le même titre qu'une célèbre nouvelle de Kipling, "Amour-des-femmes ${ }^{16}$ ». Au sein d'un récit second est relatée la déchéance d'un soldat de l'armée des Indes, Larry Tighe, amant volage rongé par la syphilis et par le remords. Dans le reportage, le portrait de Mancini est brossé progressivement à la faveur d'une série de petites touches. Danseur émérite et séducteur doté d'un réel charisme auprès des femmes, il est dépeint comme "un ténébreux gigolo au regard fascinateur et aux jambes délurées ${ }^{17}$ ». En inscrivant le portrait de Mancini en regard d'un modèle littéraire, Mac Orlan lève partiellement l'énigme qui entoure l'assassinat de Violette Kaye. L'intertexte incite le lecteur à confronter la nouvelle et le reportage pour imaginer les mobiles à l'origine du crime. Il exerce une action catalytique sur la lecture, dans la mesure où il donne une impulsion déterminante à l'enquête, à la recherche des hypothèses.

$10 \mathrm{Au}$ sein du récit, une intervention métatextuelle apporte quelques précisions sur l'utilité de cet intertexte. Elle engage le lecteur à chercher une explication au crime du côté des déchirures passionnelles : «Si j'ai donné à ces quelques lignes un titre qui est celui d'une des plus émouvantes nouvelles de Kipling, c'est que Mancini, comme Amour-des-femmes, le héros du grand écrivain anglais, vivait des femmes et pour la femme. Il savait créer le banal et furieux décor de ce que l'on nomme populairement: la passion ${ }^{18}$ ». Emporté par une passion forte, vitale autant que fatale, et pourvu des traits du héros ténébreux, Mancini est entraîné dans l'univers des figures littéraires. Les frontières pourtant étanches entre le monde observé et la fiction sont rendues poreuses. Comme l'écrit Christine Montalbetti à propos du récit de voyage, «le voyageur ne se contente plus d'utiliser la bibliothèque fictionnelle comme grille risquée d'interprétation du monde : il prend le réel pour la fable $[. . .]^{19}$ ".

11 Enfin, Mac Orlan s'emploie à convaincre le lecteur des potentialités littéraires propres aux sujets de ses reportages. Comme le montre le récit du piratage des cargos chargés de spiritueux, ce sont les situations riches en phénomènes inouïs et exceptionnels qui se prêtent à des transpositions fictionnelles : «La littérature s'est emparée de ce sujet. Il y a là toutes les pièces d'un roman parfaitement attrayant ${ }^{20} »$. De même, le parcours atypique du légionnaire Klems, qui, après avoir déserté, réorganisa l'artillerie d'Abd-el- 
Krim, est-il susceptible de nourrir l'affabulation d'un roman d'aventures : «L'aventure de Klems est une de celles qui, malgré tout, donnent à la Légion son pouvoir littéraire ${ }^{21}$ ». Mac Orlan motive ainsi sa propre pratique littéraire fondée sur l'exploitation de la matière figurative des récits factuels.

Le reportage, vivier de l'œuvre romanesque

Pour le romancier, le reportage fait office de vivier. Il nourrit l'invention romanesque. Il fournit des sujets, des données chiffrées, des personnages et des modèles narratifs. Il influe sur la nature de ces récits fictionnels, sur la prégnance des romans policiers et d'espionnage, deux avatars du roman d'aventures. L'action principale de Dinah Miami, le piratage du Rose-Marie II, est directement inspirée par le sujet du reportage correspondant. Quartier réservé et Le Tueur $n^{\circ} 2$ prennent appui sur l'anecdote judiciaire, sur le motif de la femme coupée en morceaux. Le Camp Domineau et La Bandera portent l'empreinte des couleurs fortes de la matière militaire. Les noms de certains personnages fictifs de Dinah Miami sont obtenus par une manipulation d'ordre paragrammatique. C'est à la faveur d'une substitution de voyelle qu'est forgé le nom du principal armateur, Kempton, sur le modèle du subrécargue Walter James Kimpton évoqué dans Les Pirates de l'avenue du Rhum.

13 En ce qui concerne l'espace, le reportage est doté d'une topographie précise qui modèle le cadre de l'action romanesque et fournit un ancrage référentiel. Le plus souvent, les notations descriptives extraites des reportages qui rappellent les territoires familiers de Mac Orlan ont l'aspect d'illustrations stéréotypées, du genre «Image d'Épinal ». Les toponymes empruntés à l'espace empirique créent un effet de couleur locale dans Le Camp Domineau, où la référence à la rue Abdallah Guèche, un quartier réservé de Tunis, s'accompagne non d'une description mais d'une immersion dans la socialité urbaine. En transposant la topographie réelle dans la géographie du roman, Mac Orlan vise à la création d'une atmosphère. Dans Le Tueur $n^{\circ} 2$, la description de la chambre de Mancini est rédigée à partir de deux séquences du reportage rapprochées, soudées et ainsi reconfigurées. Le romancier a reproduit une notation descriptive à visée sémiotique qu'il a étoffée d'un commentaire en forme de litote: "Sur les murs, des images de femmes coquettement dévêtues, qui avaient été découpées dans des journaux galants, n'apportaient point par leur présence une atmosphère de libertinage séduisant ${ }^{22} »$. Ces renseignements sont investis d'une valeur indicielle. Ils engagent le lecteur à chercher un éclairage à ces crimes sordides du côté des pathologies sexuelles. Ils font également signe par le biais du motif de la découpe. Découpe des photos de presse, découpe des corps de femme.

14 L'empreinte du reportage sur le roman est perceptible au niveau des conditions de l'acte narratif, comme au niveau du découpage et du montage des unités textuelles. Elle affecte en premier lieu l'instance narrative. Si le reporter arpente la zone située entre Wentworth Street et Petticoat Lane, en l'occurrence le territoire de Jacq l'Éventreur, aux côtés d'un "policier serviable ${ }^{23}$ ", le narrateur-personnage du roman correspondant ne se risque dans les rues du Quartier Réservé qu'en compagnie d'un inspecteur de police. Dans Quartier réservé, l'énigme est entretenue par le recours à la technique du narrateur imparfait, sur le modèle du reportage. Le narrateur commence son enquête en ne disposant que d'informations partielles ou erronées.

15 En second lieu, les romans gardent la trace d'une forme de narration simultanée déployée dans les reportages. Dinah Miami se caractérise par l'entrelacement des unités narratives. Si l'on prend l'exemple de la séquence relatant le départ de Mathieu Cauwin 
pour l'Atlantique à bord du Rose-Marie II, on remarque qu'elle alterne avec celle des préparatifs de la cantatrice Dinah-Miami embarquant dans un paquebot pour NewYork. Au sein d'une même page se croisent des événements diégétiques différents mais situés sur le même axe chronologique. Les unités narratives afférentes aux rencontres et aux agissements de Dinah Miami à New-York s'inscrivent en contrepoint de celles consacrées aux découvertes de Mathieu Cauwin à bord du Rose-Marie II. Cette structure fuguée a pour effet d'accroître le suspense et d'inciter le lecteur à combler les intervalles qui séparent chaque séquence. En menant de front deux lignes d'action différentes et parallèles dans le temps, le romancier crée un effet d'ubiquité et rend sensible la progression des périls. Inspirée de la segmentation du récit à l'œuvre dans Images sur la Tamise, cette technique de montage narratif est particulièrement appropriée au roman policier. Elle est utilisée dans Le Tueur $n^{\circ} 2$, où les enquêtes sur les assassinats de deux femmes découvertes mutilées dans des malles se déroulent au même moment, l'une en Angleterre, l'autre en Belgique.

La structure narrative du reportage assume une fonction matricielle, comme le montre, par exemple, la reprise des récits seconds, l'histoire du légionnaire Klems dans $L a$ Bandera et l'affaire du Frédéric-B, dans Dinah Miami. Tout en étant étoffé, le récit du piratage de ce cargo, fleuron de la contrebande d'alcools, est reproduit selon le même ordonnancement narratif. Dans les deux textes, ce récit enchâssé s'achève sur une chute surprenante, en forme de coup de théâtre. C'est seulement dans la clausule de ce récit qu'est révélée la véritable identité sexuelle du capitaine de ce «bateau fantôme [...] légendaire sur toute l'avenue du Rhum ${ }^{24} »$.

L'art de la greffe

17 Carnets d'enquêtes, les reportages approvisionnent les romans en savoirs qui assurent un ancrage référentiel à l'intrigue et répondent à une intention didactique. Ces reprises non explicites, littérales ou remaniées, de textes autographes ne se présentent pas sous la forme de la citation, de l'allusion ou du plagiat. Elles ressortissent plutôt à la pratique de la greffe (ou du greffage) au sens médical ou botanique du terme. Comme dans le domaine médical, l'origine du greffon reste toujours anonyme. Le greffon textuel est également susceptible d'être élagué par le romancier, de même que les horticulteurs pratiquant la greffe en couronne taillent les greffons prélevés à la fin de l'hiver. Dès lors, pour désigner les manipulations opérées sur le greffon textuel, nous proposons de forger une métaphore empruntée au lexique du jardinage. Si l'épaulement est le terme technique employé pour dénommer la taille du greffon végétal avant son insertion dans le porte-greffe, nous appellerons épaulement textuel l'opération qui consiste à remanier le greffon de telle façon qu'il s'insère opportunément dans le texte romanesque. Ces techniques de chirurgie textuelle (remodelage, réduction ou expansion des greffons, opérations suivies de leur ajustement aux spécificités de la parole romanesque) s'inscrivent parmi les opérations rédactionnelles qui ont donné naissance à La Bandera.

Gilieth, le personnage principal de $L a$ Bandera, est incorporé dans la légion étrangère espagnole de Dar Riffien. Dans le cinquième chapitre du livre, le récit de son arrivée dans le camp est interrompu par un long développement au présent de l'indicatif, tantôt itératif, tantôt atemporel, qui apporte une série d'informations sur la Légion espagnole. Ces notations précises, voire techniques, ont été partiellement prélevées sur le reportage. Celles qui déclinent l'identité des huit unités de cette Légion et, par là même, explicitent le titre du roman, sont reprises presque textuellement. Le romancier 
a néanmoins assorti les termes espagnols de leur traduction. Par delà le rituel se profilent les valeurs et la culture de ce corps d'armée :

19 La Légion étrangère espagnole est divisée en huit unités formant corps. On les appelle des banderas, du mot bannière. Elles possèdent chacune des fanions magnifiques qui correspondent à leur nom. La première bandera s'appelle Los Jabalies, ou les Sangliers, ce sont les armes de la maison de Borgona. La seconde est celle des Aquilas, les Aigles brodés sur un fond de soie pourpre. La troisième est la bandera d'El Tigre, le tigre. La quatrième, au fanion splendide est celle d'El Christo y la Virgen, la bandera du Christ et de la Vierge. La cinquième, appelée Gran Capitan (grand capitaine) est dédiée à Gonzalvo de Cordoba. La sixième est celle du Duque de Alba. La septième, dont le lieutenant-colonel fut tué à Melilla s'appelle: La Valenzuela. Le huitième et dernière porte le nom de Christophe Colomb. ${ }^{25}$

Mais, dans l'ensemble, ces notations ont été réagencées, et, pour la plupart, réécrites avant d'être raccordées au récit. Elles occupent quarante-six pages dans Légionnaires, seulement sept dans La Bandera. Le travail d'épaulement combine une double opération de sélection et d'émondage du texte-source. Ainsi sont effacés tous les témoignages de légionnaires rencontrés lors du séjour de Mac Orlan à Dar Riffien, toutes les marques de subjectivité énonciative (souvenirs et jugements), toutes les anecdotes, comme tous les propos rapportés. Le romancier procède à une synthèse d'éléments épars et parfois récurrents (comme, par exemple, les notations relatives à la solde des légionnaires), qu'il regroupe dans quatre longs paragraphes, dotés d'une forte cohérence thématique. Le lecteur est successivement instruit de la mission de cette unité militaire, des motivations des légionnaires, des différences entre les Légions étrangères française et espagnole et des signes distinctifs propres à chaque unité. Le lissage du texte originel confère à cette séquence l'allure d'un développement historico - sociologique qui ne surprend pas le lecteur, ne crée pas un phénomène de rejet, en raison de la préparation $\mathrm{du}$ porte-greffe. Une liaison thématique ${ }^{26}$ assure en effet la soudure entre cette séquence didactique et le récit de l'installation de Gilieth : « Gilieth prit rapidement le ton et le caractère du corps. La Légion étrangère de Dar Riffien constitue une troupe solide, parfaitement entraînée et qui sait mourir au feu [...] ${ }^{27}$ ».

21 À l'épaulement s'ajoute une autre pratique d'écriture qui se rapproche de la marqueterie, dans la mesure où elle est fondée sur la recomposition de fragments de greffon. Dans La Bandera, le portrait très succinct du commandant de la Légion, le colonel Millan Astray, est le fruit d'un double remaniement textuel. Il résulte d'un processus de soudure et de permutation qui prend appui sur trois unités textuelles $\mathrm{du}$ reportage. Nous proposons donc de confronter le texte-source et le texte-cible. Dans Légionnaires, le reporter exprime l'admiration que lui inspire «cet homme, droit et maigre, mutilé par de nombreuses blessures, [qui] avait su imposer sa personnalité à cette jeune légion [...]». Dans le chapitre suivant affleure un nouveau croquis. Millan Astray « est un homme grand et mince, à courtes moustaches. Il n'a plus qu'un œil et qu'un bras ». Quelques pages plus loin, le portrait des jeunes officiers de la Légion espagnole est précédé d'une médiation culturelle, d'un filtre pictural: "Les jeunes capitaines du Tercio ne sont pas très différents des figures madrilènes peintes par Velasque $z^{28} »$. Au terme d'un travail de prélèvement, de permutation et de soudure s'esquisse le portrait du commandant de la Légion :

[ c'est à Beni Hosmar] que son colonel Millan Astray perdit un œil, avant de perdre un bras quelques mois plus tard. Aussi la silhouette maigre et énergique de Millan Astray 
est-elle inoubliable. Tels étaient peints, par Vélasquez, les grands capitaines castillans à l'époque où l'infanterie espagnole était la plus célèbre dans le monde. ${ }^{29}$

La mise en rapport du personnage authentique avec la figure picturale du grand capitaine rappelle le thème proustien de la confusion entre l'art et la réalité. Cette interaction anoblit et transfigure le personnage réel, lui confère les qualités de l'œuvre d'art.

Les développements historico-sociologiques intégrés dans les romans résultent parfois de la coalescence de plusieurs micro-unités disséminées dans les reportages. Le cinquième chapitre du Camp Domineau s'ouvre sur un paragraphe qui associe l'historique du bataillon disciplinaire d'Afrique à des informations géographiques afférentes à la répartition des compagnies dans le sud tunisien : « Le roi Louis-Philippe, en 1831, créa la Légion étrangère et en 1832 l'Infanterie légère d'Afrique. Ces deux corps très différents et par leur origine et par leur esprit ne s'aiment pas. La Légion, particulièrement, tient les Joyeux en dehors du cercle formé par ses relations ${ }^{30}$ ». Ce segment textuel à visée documentaire est reconstitué à partir de plusieurs informations aimantées et réticulées. Le parallèle établi entre la Légion et le bataillon disciplinaire est destiné à un lectorat peu avisé des spécificités de chaque corps. Il est absent du reportage.

Mais les refigurations de l'intertexte journalistique ne sont pas réductibles à des processus de condensation, de déplacement ou de fusion. Elles donnent également lieu à des expansions, terme synthétique recouvrant des phénomènes d'addition textuelle, d'accentuation et de dissémination. Un motif accessoire des Pirates de l'avenue du rhum, la présence virtuelle de "bateaux-dancings" à la limite des eaux territoriales américaines, est développé dans Dinah Miami. Il sert de support à un effet de complication de l'intrigue et motive l'insertion, en italiques, du texte de la chanson interprétée par Dinah Miami. Compositeur lui-même, Mac Orlan a su tirer parti des ressources expressives propres à l'inclusion de la chanson dans le texte littéraire. La plupart de ses romans sont émaillés de chansons. Le motif du "bateau-dancing » a donc été exploité par le romancier car il se prête à des combinatoires textuelles, motive l'entrecroisement de modes d'expression hétérogènes. L'ajustement du greffon aux propriétés du tissu romanesque est sensible dans les modalités d'insertion de l'exposé informatif, comme dans les changements de construction énonciative et de perspective narrative. L'intégration du greffon au tissu fictionnel varie en fonction de sa nature. Les séquences narratives ou descriptives sont insérées dans la trame romanesque par une liaison thématique. En revanche, les séquences informatives sont le plus souvent situées en tête de chapitre afin de ne pas interrompre la coulée narrative. Fruit d'une condensation d'éléments épars dans le reportage, l'exposé socio-linguistique ${ }^{31}$ qui ouvre le chapitre neuf de Dinah Miami se signale par l'emploi d'un déictique spatial et $d u$ présent itératif. La mise en page propre à l'ouverture du chapitre fait également office de signal démarcatif. Tous ces procédés, qui répondent à une recherche de sobriété et de discrétion, sont dictés par le souci de privilégier la dynamique narrative. Dans ces romans, l'intérêt dramatique prime toujours sur l'intérêt documentaire.

Enfin, l'ajustement du greffon au texte fictionnel revêt l'aspect de transmodalisations, lesquelles s'expliquent par la prégnance des catégories de la voix et du mode, techniques modales d'organisation du récit. Dans Le Bataillon de la mauvaise chance, la distinction des deux types de sous-officiers est insérée dans un exposé didactique à la troisième personne, alors que, dans Le Camp Domineau, elle est attribuée à un 
personnage, le bataillonnaire François Mutche, qui répond à l'interrogation d'un officier italien peu averti de la signification des tenues vestimentaires: "Cadre noir, c'est-à-dire sorti des rangs du bataillon, cadre blanc, venu des réguliers, de l'extérieur. C'est à cause de l'ancien uniforme des Joyeux que ces noms ont été choisis ${ }^{32}$ ». L'exposé du savoir est fréquemment confié à un protagoniste du récit. Comme l'a montré Philippe Hamon, dans Le Personnel du roman, l'énoncé didactique, ainsi motivé, ne perturbe plus la lecture du récit. De surcroît, l'information narrative prélevée sur le reportage est remaniée, régulée en fonction du système modal dominant. Dans Dinah Miami, l'épisode du piratage du Rose-Marie II est raconté selon le point de vue limité de Mathieu Cauwin, qui n'a qu'une perception partielle et confuse de l'agression perpétrée contre le cargo. Cette adaptation du greffon au porte-greffe ménage des effets d'angoisse et de suspens. Elle préserve l'intérêt dramatique de l'épisode en raison des rebondissements que le lecteur découvre en même temps que le personnage.

$\mathrm{Au}$ moment de la rédaction de ses récits fictionnels, Mac Orlan relit et manipule ses reportages qui lui servent de documents préparatoires. Ces récits factuels sont étayés par un double intertexte, factuel et littéraire, lequel modèle la perception du monde jusqu'à la brouiller lorsque fusionnent les espaces réels et fictionnels. Éléments constitutifs de la genèse des romans, ces dossiers documentaires affectent sensiblement leur nature. L'interaction des régimes factuel et fictionnel du récit retentit sur la portée didactique des romans, comme sur la spectacularisation de l'énoncé. De surcroît, la transplantation textuelle pratiquée par Mac Orlan donne naissance à une zone d'intersection entre le discours référentiel et le discours fictionnel, un troisième pôle dénommé «référencialité» par Tiphaine Samoyault. Ce néologisme désigne le phénomène de "référence de la littérature au réel [...] médiée par la référence proprement intertextuelle ${ }^{33} »$. Il s'applique au type d'opérations régissant les rapports entre le texte littéraire et le référent réel, à la différence que, dans les œuvres romanesques mac orlaniennes inspirées des reportages, la référence au réel est doublement médiatisée : l'intratexte greffé sur le tissu fictionnel est lui-même fécondé par des références littéraires.

Le parcours critique qui mène du reportage au roman nous procure quelques aperçus sur la naissance et les métamorphoses de l'écriture romanesque. L'exploration des modes d'intertextualité externe et interne nous conduit jusque dans les coulisses de la création, au croisement des deux notions forgées par Raymonde Debray-Genette, l'exogenèse (l'inscription des éléments préparatoires) et l'endogenèse ( « la coalescence, l'interférence et la structuration des seuls constituants de l'écriture $\left.{ }^{34} »\right)$. Ainsi apparaissent en pleine lumière les desseins et les gestes de l'écrivain, les processus formels qui président au recyclage des textes journalistiques. Épaulement, redéploiement, expansion et ajustement, telles sont les quatre principales catégories qui régissent la pratique de la greffe, le remaniement de l'intertexte. Le roman impose sa forme (ses codes, ses ressources et ses configurations textuelles) aux formes empruntées au discours sur le réel. 


\section{NOTES}

1. Nous nous référerons à cinq reportages publiés entre 1925 et 1984 (publication posthume) : Les Pirates de l'avenue du Rhum, Paris, éditions du Sagittaire, 1925 ( abréviation utilisée : PAR) ; Images sur la Tamise, Paris, éditions du Sagittaire, 1925 (abréviation utilisée : IT) ; Légionnaires, Paris, éditions du Capitole, 1930, (abréviation utilisée : L) ; Le bataillon de la mauvaise chance, Paris, Les éditions de France, 1933 (abréviation utilisée : BMC) ; Le Mystère de la Malle $n^{\circ} 1$, édition de Francis Lacassin, Paris, U.G.E., coll. 10/18, 1984 (abréviation utilisée : MM1).

2. Les cinq romans de Mac Orlan seront désignés à l'aide des sigles suivants placés entre parenthèses, à la suite des références éditoriales : Dinah Miami, roman inséré dans $L a$ Vénus internationale, Gallimard, coll. Folio, $\mathrm{n}^{\circ} 1329,(D M)$; La Bandera, Gallimard, coll. Folio, $n^{\circ} 244,(B)$; Quartier réservé, Gallimard, coll. Folio, $n^{\circ} 2584(Q R)$; Le Tueur $n^{\circ} 2$, Gallimard, coll. «L'imaginaire », n 264 (TN2) ; Le Camp Domineau, Gallimard, coll. Folio, $\mathrm{n}^{\circ} 2459$ ( $\left.C D\right)$.

3. Pour Bernard Baritaud, son meilleur exégète, le journaliste a collecté des « choses vues » dans le dessein d'alimenter la forge du romancier : « Les romans de Mac Orlan sont souvent précédés de reportages préparatoires, et leur auteur se considérait alors plus comme "un écrivain en promenade d'étude" que comme un véritable journaliste ". Bernard Baritaud, Pierre Mac Orlan, sa vie, son temps, Genève, Droz, 1992, p. 225.

4. Terme utilisé notamment par Tzvetan Todorov dans Symbolisme et interprétation, Paris, Seuil, 1978, p. 61-62.

5. Gérard Genette, Fiction et diction, Paris, Seuil, 1991, p. 93.

6. PAR, p. 37.

7. Michel Collomb, La littérature art déco, Paris, Klincksieck, 1987, p. 208.

8. PAR, p. 64.

9. Ibid., p. $19-20$ et p. 24.

10. «Dans le reportage, écrit Michel Collomb, le fait est représenté dans son individualité irréductible et concrète. Le cas particulier qui est ainsi représenté n'est dès lors qu'un exemple, qu'une illustration d'une situation générale [...] », op. cit., p. 207. 11. PAR, p. 10-11.

12. Christine Montalbetti distingue les relations de médiation, de comparaison, d'inclusion et de coïncidence. Voir son livre Le Voyage, le monde et la bibliothèque, Paris, P.U.F., 1997 et son article théorique « Entre écriture du monde et récriture de la bibliothèque » paru dans Miroirs de textes, Nice, Association des publications de la Faculté des lettres, 1998, p. 15-16.

13. BMC, p. 56.

14. Ibid., p. 57.

15. Ibid., p. 60.

16. Cette nouvelle a été publiée pour la première fois en 1893, dans le recueil Many inventions (titre français : Tours et détours). Elle figure dans le volume II des Euvres complètes de Rudyard Kipling, publiées dans la collection de la Pléiade par les éditions Gallimard, (édition de Pierre Coustillas et Marie-Claire Hamard) sous le titre « Chérides-dames », p. 199-224.

17. MM1, p. 29.

18. Ibid., p. 29-30. 
19. Christine Montalbetti, «Entre écriture du monde et récriture de la bibliothèque ", in Miroirs de textes, op. cit., p. 11.

20. PAR, p. 53-54.

21. BMC, p. 128.

22. TN2, p. 71.

23. IT, p. 75.

24. DM, p. 276.

25. B, p. 55-56.

26. Nous donnons à ce terme l'acception que lui prête Marcel Cressot. Il entend par liaison thématique « la mise en rapport, grâce à une place privilégiée, d'un terme avec un autre terme précédent et qui représente la même idée ou une idée analogue ", in Le Style et ses techniques, Paris, P.U.F., 13 ème édition, 1991, p. 241.

27. B, p. 54.

28. $L$, p. $20,51,55$.

29. $B$, p. 55.

30. $C D$, p. 61.

31. Mac Orlan traduit tous les termes anglais qui désignent les divers protagonistes de la contrebande d'alcools, tels les high-jackers.

32. Ibid., p. 223.

33. Tiphaine Samoyault, L'Intertextualité, mémoire de la littérature, Paris, Nathan Université, coll. 128, 2001, p. 83.

34. Raymonde Debray-Genette, "Génétique et poétique : le cas Flaubert », in Essais de critique génétique, Paris, Flammarion, 1979, p. 67.

\section{RÉSUMÉS}

Journaliste pendant l'entre-deux-guerres, Pierre Mac Orlan a notamment écrit cinq reportages qui lui ont servi de document préparatoire pour sa création romanesque. Résultats d'une investigation ou d'une plongée dans un monde peu connu du grand public, ces discours sur le monde sont infiltrés par deux formes de pratiques intertextuelles (la citation et la référence). L'intertexte factuel y assume une double fonction, pragmatique et argumentative. Quant à l'intertexte littéraire, il brouille la représentation de la chose vue à la faveur d'une relation d'interférence, laquelle a pour fondement la remise en question de l'hétérogénéité du texte littéraire et de la réalité sensible. Sur le plan de la poétique romanesque, les reportages assument une double fonction, matricielle et nutritive. Ils approvisionnent les récits fictionnels en sujets, structures et personnages. Ils fournissent également des greffons transplantés dans le corps romanesque au terme de quatre opérations textuelles: l'épaulement, le redéploiement, l'expansion et l'ajustement. En définitive, cette pratique intertextuelle nous invite, d'une part, à redéfinir les rapports entre récit factuel et récit fictionnel, et, d'autre part, à interroger les processus rédactionnels à l'œuvre dans l'une des phases de la genèse romanesque. 
INDEX

Index chronologique : XXe siècle

Mots-clés : Pierre Mac Orlan 